\title{
Fibronectin-mediated inflammatory signaling through integrin $\alpha 5$ in vascular remodeling
}

\author{
Madhusudhan Budatha ${ }^{1,2,3^{*}}$, Jiasheng Zhang ${ }^{2}$, Martin A. Schwartz ${ }^{2,3,4}$
}

${ }^{1}$ Department of Medicine, Division of Nephrology, University of Texas Long School of Medicine, San

Antonio, TX 78229, USA

${ }^{2}$ Yale Cardiovascular Research Center, 300 George Street, New Haven, CT 06511, USA

${ }^{3}$ Department of Internal Medicine (Cardiology), Yale School of Medicine, New Haven CT

${ }^{4}$ Departments of Cell Biology and Biomedical Engineering, Yale University, New Haven CT

Running Title: Integrin $\alpha 5$ in pathological vascular remodeling

*Corresponding author: budatha@uthscsa.edu 


\begin{abstract}
:
Adhesion of vascular endothelial cells (ECs) to the underlying basement membrane potently modulates EC inflammatory activation. The normal basement membrane proteins laminin and collagen IV attenuate inflammatory signaling in part through integrin $\alpha 2 \beta 1$. In contrast, fibronectin, the provisional matrix protein found in injured, remodeling or inflamed vessels, sensitizes ECs to inflammatory stimuli through integrins $\alpha 5 \beta 1$ and and $\alpha v \beta 3$. A chimeric integrin in which the cytoplasmic domain of $\alpha 5$ is replaced with that of $\alpha 2$ pairs with $\beta 1$ and binds fibronectin but signals like $\alpha 2 \beta 1$. Here, we examined mice in which integrin $\alpha 5$ is replaced with the $\alpha 5 / 2$ chimera, using the transverse aortic constriction (TAC) and partial carotid ligation (PCL) models of vessel remodeling. Following TAC and PCL surgery, WT mice showed increased fibronectin deposition and expression of inflammatory markers, which were strongly attenuated in a5/2 mice. $\alpha 5 / 2$ mice also showed reduced artery wall hypertrophy in the TAC model and diminished inward remodeling in the PCL model. Acute atherosclerosis after PCL in hyperlipidemic ApoE-/- mice on a high fat diet was dramatically decreased in $\alpha 5 / 2$ mice. These results underscore the key role for integrin $\alpha 5$ signaling in pathological vascular remodeling and support its potential as a therapeutic target.
\end{abstract}




\section{Introduction:}

Arteries remodel in response to physiological stresses such as changes in blood pressure or fluid shear stress, and to pathological stresses such as low and disturbed shear stress, hyperlipidemia, diabetes or oxidative stress among others $^{1,2}$. Physiological remodeling is characterized by homeostasis, in which key variables return to their original levels or set point after a perturbation 1,3,4. For example, increases in blood pressure induce hypertrophic thickening of the vessel wall to restore tensile wall stress to close to original values. Increased or decreased blood flow through a vessel with concomitant changes in fluid shear stress results in outward or inward remodeling of vessel diameters to restore shear stress to close to original values. Inflammation is an essential component of all remodeling ${ }^{5}$. Cells of the vascular wall that register changes in homeostatic variables (wall or fluid shear stress) activate inflammatory pathways, resulting in recruitment of leukocytes, most prominently monocytes. These cells contribute to these processes by extracellular matrix (ECM) production and degradation, and cytokine/chemokine secretion. Restoration of wall or fluid shear stress to homeostatic levels leads to suppression of inflammatory signaling and restoration of the normal state.

Pathological remodeling, exemplified by atherosclerotic lesions, resembles physiological remodeling in many respects but differs in that the initiating stimuli are never terminated and the inflamed state never resolved ${ }^{4,6}$. But ECM remodeling is a central element of both physiological and pathological processes ${ }^{6,7}$. Vascular remodeling is universally accompanied by expression and assembly of provisional ECM proteins such as fibronectin (FN), thrombospondin or fibrin, whereas vessel stabilization and quiescence are associated with loss of these components and assembly of basement membranes where collagen IV (coll IV) and laminin (lam) are the main protein

components ${ }^{8,9}$. Cells interact with provisional ECM proteins through RGD-binding integrins, of which integrins $\alpha 5 \beta 1$ and $\alpha v \beta 3$ are the most prominent, whereas RGD-independent integrins $\alpha 2 \beta 1, \alpha 6 \beta 1$ and $\alpha 6 \beta 4$ are the main receptors for collagens and laminins ${ }^{10}$.

Importantly, ECM remodeling also modulates inflammatory activation. In endothelial cells, lam/coll IV basement membranes limit inflammatory responses through signaling by integrin $\alpha 2 \beta 1$, while FN enhances responses to inflammatory stimuli, including disturbed fluid shear stress, IL1-b and oxidized LDL through integrin $\alpha 5 \beta 1$ and 
$\alpha v \beta 3{ }^{9}$. Though less studied, coll/lam basement membranes also promote smooth muscle cell (SMC) quiescence and differentiation ${ }^{11,12}$. The effects of these different ECM proteins were traced to the integrin $\alpha$ subunit cytoplasmic domains: a chimeric integrin in which the $\alpha 5$ cytoplasmic domain was replaced with that of $\alpha 2$ binds fibronectin and supports cell adhesion and cytoskeletal organization normally but signals as if the cells were on lam or coll ${ }^{12}$. A mouse in which this mutation was inserted into the $\alpha 5$ locus developed normally and is healthy and fertile but showed reduced endothelial inflammatory marker expression in regions of disturbed flow, developed smaller and less inflamed atherosclerotic lesions in hyperlipidemia, and showed improved recovery from hindlimb ischemia $^{12,13}$.

In the current study, we analyzed integrin $\alpha 5 / 2$ mice in three new models of vascular remodeling. We report that these mice show dramatic decreases in inflammation and remodeling, both physiological and pathological.

\section{Results}

Transverse aortic constriction (TAC)

To assess the role of integrin $\alpha 5$ signaling in pressure-induced artery remodeling, we subjected mice to TAC or to sham surgery as a control. This was accomplished by placing a clamp between the innominate artery and the left carotid, a well-established experimental method to increase pressure in the right carotid artery ${ }^{14-16}$ (Figure 1A). Left and right carotid arteries were collected one week post-surgery. We observed significant increases in vessel area and lumen diameter of the right carotid artery compared with sham, or with the contralateral left carotid (Figure 1B,C). These changes were accompanied by strongly elevated fibronectin deposition in the endothelial layers of right carotids but not in contralateral left carotids and sham carotids (Figure 1D).

\section{Transverse aortic constriction in $\alpha 5 / 2$ mice}

To investigate the role of fibronectin-integrin $\alpha 5$ signaling in TAC-induced structural remodeling of the carotid arteries, we compared these results to integrin $\alpha 5 / 2$ knock-in mice. Right carotids from $\alpha 5 / 2$ knock in mice enlarged less than WT mice (Figure 2A,B). The accumulation of FN seen in WT mice was largely suppressed in $\alpha 5 / 2$ carotids (Figure 3A). Staining for the leukocyte adhesion receptors and inflammatory markers VCAM1 and 
ICAM1 showed strong elevation in the endothelium of the right carotid in WT mice but only minor changes in $\alpha 5 / 2$ mice (Figure 3B,C). Staining for CD68 as a marker for monocyte/macrophages showed strong inflammatory cell recruitment to the right carotid from WT mice, but only small changes in $\alpha 5 / 2$ mice (Figure 3D). Finally, we stained these tissues for pS536 NF-KB to assess activation of this critical inflammatory transcription factor. TAC induced strong NF- $\kappa \mathrm{B}$ activation in the right carotid from WT mice but no significant changes in $\alpha 5 / 2$ mice (Figure 3E). Left carotids from both genotypes showed only minor changes in any of these markers. Together, these data show that inflammation and structural remodeling in this model of acute hypertension are drastically reduced in $\alpha 5 / 2$ chimera mice, consistent with a role of integrin $\alpha 5$

\section{Partial carotid ligation.}

Partial carotid ligation in mice decreases blood flow magnitude and introduces disturbances into the flow patterns in the common carotid, resulting in inflammatory activation of the endothelium and reduced lumen volume ${ }^{17,18}$. We first subjected WT mice to PCL surgery and examined carotid arteries at one-week post-surgery (Figure 4A). PCL resulted in reduced lumen diameter of the left carotid compared to the right carotid (Figure 4B). We also observed a dramatic increase in FN staining in the endothelial layer of the left but not right carotid arteries (Figure 4C). These observations prompted us to test the involvement of integrin $\alpha 5$ in these events by performing PCL in $\alpha 5 / 2$ mice. In contrast to WT mice, integrin $\alpha 5 / 2$ mice showed no change in the lumen area (Figure 4B). Further, ICAM1 and VCAM1 were strongly induced in the left carotid in WT mice but changed little in $\alpha 5 / 2$ mice (Figure 5A,B). Consistent with this finding, inflammatory cell recruitment marked by CD45 and CD68 was also attenuated in the left carotid of $\alpha 5 / 2$ mice (Fig. 5C.D) as was activation of NF- $\kappa B$ (Figure 5E). The contralateral right carotids of WT and $\alpha 5 / 2$ knock-in mice remained negative. PCL-induced artery inflammation and structural remodeling thus depend strongly on FN signaling through integrin $\alpha 5$.

\section{Acute atherosclerosis after PCL in hypercholesterolemic $\mathrm{ApoE}^{-/-}$mice}

PCL in hypercholesterolemic mice is used to model acute disturbed shear-induced atherosclerosis ${ }^{19,20}$. Lesions develop within a few weeks due to the very low and strongly oscillatory shear stress induced by surgery. To test the role of integrin $\alpha 5$ in this model, we crossed heterozygous $\alpha 5 / 2$ knock in mice with hypercholesterolemic 
ApoE $^{-/-}$mice (Jackson laboratories, B6.129p2-Apoetm1Unc/j; Cat\#002052) to generated WT;ApoE-/- and a5/2;ApoE ${ }^{-/-}$mice. Mice at 8-10 weeks were subject to PCL and fed a Western diet (RD Western diet \#D12079B, Open Source Diet) for 3 weeks. H\&E staining demonstrated significant atherosclerosis in the LCA of WT; ApoE ${ }^{-/-}$ mice whereas plaque in $\alpha 5 / 2 ; \mathrm{ApoE}^{-/-}$mice was barely detected (Figure 6A). Staining lipids with Oil Red O confirmed the reduction in plaque in $\alpha 5 / 2$ mice (Figure 6B). Monocytes/macrophage (CD68 ${ }^{+}$cells) infiltration in the LCA was also markedly reduced in $\alpha 5 / 2 ; \mathrm{ApoE}^{-/-}$mice (Figure $6 \mathrm{C}$ ). As before, the contralateral side was unaffected and showed negligible plaque formation on this time scale. Plasma lipid profiles of total cholesterol, LDL cholesterol and triglycerides were not statistically different between WT and $\alpha 5 / 2$ mice (Figure 7). Overall, these results revealed that the $\alpha 5 / 2$ knock in strongly attenuated flow-dependent acute atherosclerosis.

\section{Discussion:}

In this study, we report that the integrin $\alpha 5 / 2$ mutation in mice. which abolishes the inflammatory effects of FN, strongly affects vascular remodeling. In the TAC model of acute hypertension in the right carotid artery, $\alpha 5 / 2$ mice show markedly less FN deposition, less inflammatory activation of the endothelium, and less artery wall thickening. It should be noted that $\mathrm{C} 57 \mathrm{Bl} / 6$ mice show excessive adventitial thickening in hypertension, perhaps related to the susceptibility of this strain to inflammatory stimuli ${ }^{21,22}$. These responses may thus be regarded as partially pathological in that remodeling overshoots the homeostatic goal. This effect was abolished by the $\alpha 5 / 2$ mutation, consistent with its ability to limit inflammation.

Partial carotid ligation is principally a model of flow-induced remodeling due to induction of low and oscillatory flow in the affected common carotid artery. Altered flow leads to inflammatory activation of the endothelium and inward remodeling to reduce lumen diameter, sometimes to near closure, a feature more in keeping with pathological than physiological processes, likely due to the oscillatory flow component. Consistent with high FN deposition in the endothelial layer of WT mice, inflammatory activation and inward remodeling were strongly blunted in $\alpha 5 / 2$ mice. To expand on these findings, we then examined effects of PCL in hypercholesterolemic mice, a model of acute flow-induced atherosclerotic plaque formation. Plaque size, lipid accumulation and 
inflammatory cell recruitment were all greatly decreased. These results strongly support a major role for inflammatory FN signaling in disturbed flow-induced vessel pathology.

Vascular remodeling in response to altered mechanical forces derived from blood flow and pressure is physiological when key variables such as tensional wall stress or fluid shear stress are restored to close to their initial levels ${ }^{2,3}$. Physiological remodeling requires inflammation and participation of immune cells but is carefully regulated and resolves when key variables return to their initial value or set point. By contrast, remodeling is pathological when initial set points are not restored due to either insufficiency or, more often, to overcompensation. For example, artery walls in hypertension thicken too much and lose elasticity, which exacerbates hypertension and its consequences for the microvasculature. Importantly, excessive or uncontrolled inflammation is the principle cause of pathological remodeling, typified by formation of atherosclerotic lesions in regions of low/disturbed shear stress ${ }^{23}$. Adventitial fibrosis and stiffening in hypertension provides another example ${ }^{22}$.

The current results identify FN and integrin $\alpha 5$ signaling as key elements of pathological vascular remodeling in acute models of both hypertension and disturbed flow. We note that mice in these experiments were total knockins, thus many cell types may contribute to the observed effects. Assessing effects in cell-type specific conditional knock-ins is an important direction for future work. While the ultimate goal of these studies is the identification of therapeutic targets, FN or integrin $\alpha 5$ themselves are essential for physiological processes and thus seem unlikely candidates for direct intervention. However, elements of downstream pathways have been discovered, including the interaction of phosphodiesterase 4D (PDE4D) with the $\alpha 5$ cytoplasmic domain and the interaction of PDE4D with the $\mathrm{B} 55$ subunit of protein phosphatase $2 \mathrm{~A}^{24,25}$. Blocking these downstream pathways may be more promising avenues for translation, as are further investigations into mechanisms of a5 inflammatory signaling in atherosclerosis and hypertension. 


\section{Methods:}

Partial carotid ligation model: 10-12 weeks old C57BL6, $\alpha 5 / 2$ integrin knock-in mice and ApoE ${ }^{-/-}$mice were used for all animal studies. All the mice experiments were performed according to the approved protocol of the IACUC at Yale University. Mice were anaesthetized with ketamine and xylazine and surgery was performed. Briefly, 3 out of 4 branches of the left common carotid artery (left external carotid, internal carotid, and occipital artery) were ligated with suture, while superior thyroid artery was left intact. Mice sacrificed after isoflurane inhalation, perfused and vascular tree isolated along with carotid arteries at the indicated time points.

Transverse aortic constriction: 10-12 weeks old mice anaesthetized with ketamine and xylazine and hair was removed. A Para median incision was introduced with $1 \mathrm{~mm}$ sternum and each rib was cut individually, to expose heart and thymus, which was retracted to expose the aorta and carotid arteries. A curved 22-gauze needle with 9-0 sutures passed under aorta between carotid arteries and 27-gauze needle, space was placed on the arch prior to securing the ligature. The space was then removed, thus leaving a desired stenosis. The chest was closed with 3 to 5 simple interrupted sutures (5.0) followed by skin closure. Buprenorphine was given subcutaneously at $8 \mathrm{~h}$ interval following surgery. Mice sacrificed after isoflurane inhalation, perfused, and vascular tree isolated along with carotid arteries at indicated times.

Immunofluorescence and histochemistry: Carotid arteries embedded in optimal cutting temperature, frozen on dry ice and stored at $-80^{\circ} \mathrm{C}$. Carotid arteries were sectioned on cryostat to generate $10-15 \mathrm{~m}$ sections. Cryosections were fixed in acetone for $10 \mathrm{~min}$ at $-20^{\circ} \mathrm{C}$, blocked in IHC Tek antibody diluent for 1 hour at room temperature, and incubated with indicated antibodies in IHC Tek antibody diluent buffer. Antibodies were phosphor-NFк $\beta$-P65; FN (1:400; Sigma); VCAM1 (1:200 BD Bioscience); ICAM1 (1:200, Biolegend); CD68 (1:200, Abcam); CD45 (1:200, BD Biosciences); Sections were washed 3 times with PBS and incubated with Alexa flour 598-conjugated donkey anti rabbit or rat secondary antibody for 1 hour at room temperature. Slides were washed with PBS and mounted in Vectashield with DAPI. Images were acquired using Nikon 4 laser confocal microscope. 
Quantification and Statistical Analysis: NIH Image J program was used for quantification. Lumen diameter, vessel area, staining area was calculated from carotid artery sections. All graphs were created using GraphPad Prism software (San Diego, CA). Statistical analysis were performed using a one-way ANOVA with Turkeys post hoc analysis; $\mathrm{p}<0.05$ was considered statistically significant. All data are expressed as mean $\pm \mathrm{SEM}$

Acknowledgements: Lipid analysis was done by the Yale Mouse Phenotypic Center. This work was supported by National Institute of Health (RO1 HL75092) to MAS.

Author's contribution: MB designed the study, performed all experiments, and analyzed data and wrote the article. MB performed most of the studies at Yale and completed the analysis at University of Texas Health Sciences, San Antonio. JZ performed the mouse surgeries. MAS initiated the project, co-wrote the article, and provided financial support. 


\section{References:}

1. Chien S. Mechanotransduction and endothelial cell homeostasis: The wisdom of the cell. Am J Physiol Heart Circ Physiol. 2007;292:H1209-1224

2. Humphrey JD. Mechanisms of arterial remodeling in hypertension: Coupled roles of wall shear and intramural stress. Hypertension. 2008;52:195-200

3. Humphrey JD. Mechanisms of vascular remodeling in hypertension. Am J Hypertens. 2020

4. Baeyens N, Bandyopadhyay C, Coon BG, Yun S, Schwartz MA. Endothelial fluid shear stress sensing in vascular health and disease. J Clin Invest. 2016;126:821-828

5. Medzhitov R. Origin and physiological roles of inflammation. Nature. 2008;454:428-435

6. Libby P, Theroux P. Pathophysiology of coronary artery disease. Circulation. 2005;111:3481-3488

7. Astrof S, Hynes RO. Fibronectins in vascular morphogenesis. Angiogenesis. 2009;12:165-175

8. Finney AC, Stokes $\mathrm{KY}$, Pattillo $\mathrm{CB}$, Orr AW. Integrin signaling in atherosclerosis. Cell Mol Life Sci. $2017 ; 74: 2263-2282$

9. Yurdagul A, Jr., Orr AW. Blood brothers: Hemodynamics and cell-matrix interactions in endothelial function. Antioxid Redox Signal. 2016;25:415-434

10. Hynes RO. Integrins: Bidirectional, allosteric signaling machines. Cell. 2002;110:673-687

11. Orr AW, Lee MY, Lemmon JA, Yurdagul A, Jr., Gomez MF, Bortz PD, Wamhoff BR. Molecular mechanisms of collagen isotype-specific modulation of smooth muscle cell phenotype. Arterioscler Thromb Vasc Biol. $2009 ; 29: 225-231$

12. Orr AW, Hastings NE, Blackman BR, Wamhoff BR. Complex regulation and function of the inflammatory smooth muscle cell phenotype in atherosclerosis. J Vasc Res. 2010;47:168-180

13. Budatha M, Zhang J, Zhuang ZW, Yun S, Dahlman JE, Anderson DG, Schwartz MA. Inhibiting integrin alpha5 cytoplasmic domain signaling reduces atherosclerosis and promotes arteriogenesis. J Am Heart Assoc. 2018;7 
14. Zhang X, Feng $T$, Zeng XI, Liang $H$, Situ B, Zhang Q, Zhou F, Chen Y, Wang T, Cai D, Lin X, Xiu J, Zheng L. Identification of transcriptional variation in aortic remodeling using a murine transverse aortic constriction (tac) model. Front Cardiovasc Med. 2020;7:581362

15. Richards DA, Aronovitz MJ, Calamaras TD, Tam K, Martin GL, Liu P, Bowditch HK, Zhang P, Huggins GS, Blanton RM. Distinct phenotypes induced by three degrees of transverse aortic constriction in mice. Sci Rep. 2019;9:5844

16. Eberth JF, Gresham VC, Reddy AK, Popovic N, Wilson E, Humphrey JD. Importance of pulsatility in hypertensive carotid artery growth and remodeling. J Hypertens. 2009;27:2010-2021

17. Korshunov VA, Solomatina MA, Plekhanova OS, Parfyonova YV, Tkachuk VA, Berk BC. Plasminogen activator expression correlates with genetic differences in vascular remodeling. J Vasc Res. 2004;41:481490

18. Yuan S, Yurdagul A, Jr., Peretik JM, Alfaidi M, Al Yafeai Z, Pardue S, Kevil CG, Orr AW. Cystathionine gamma-lyase modulates flow-dependent vascular remodeling. Arterioscler Thromb Vasc Biol. $2018 ; 38: 2126-2136$

19. Nam D, Ni CW, Rezvan A, Suo J, Budzyn K, Llanos A, Harrison D, Giddens D, Jo H. Partial carotid ligation is a model of acutely induced disturbed flow, leading to rapid endothelial dysfunction and atherosclerosis. Am J Physiol Heart Circ Physiol. 2009;297:H1535-1543

20. Kumar S, Kang DW, Rezvan A, Jo H. Accelerated atherosclerosis development in c57bl6 mice by overexpressing aav-mediated pcsk9 and partial carotid ligation. Lab Invest. 2017;97:935-945

21. Taherzadeh Z, VanBavel E, de Vos J, Matlung HL, van Montfrans G, Brewster LM, Seghers L, Quax PH, Bakker EN. Strain-dependent susceptibility for hypertension in mice resides in the natural killer gene complex. Am J Physiol Heart Circ Physiol. 2010;298:H1273-1282

22. B. S, M. L, S. M, AW. C, AB. R, S-I. M, A. R, C-S. H, B. J, M. W, MR. B, G. T, JD. H. Genetic background dictates aortic fibrosis in hypertensive mice. BIORXIV. 2020 
23. Kotas ME, Medzhitov R. Homeostasis, inflammation, and disease susceptibility. Cell. 2015;160:816-827

24. Yun S, Budatha M, Dahlman JE, Coon BG, Cameron RT, Langer R, Anderson DG, Baillie G, Schwartz MA. Interaction between integrin alpha5 and pde4d regulates endothelial inflammatory signalling. Nat Cell Biol. 2016;18:1043-1053

25. Yun S, Hu R, Schwaemmle ME, Scherer AN, Zhuang Z, Koleske AJ, Pallas DC, Schwartz MA. Integrin alpha5beta1 regulates pp2a complex assembly through pde4d in atherosclerosis. J Clin Invest. 2019;129:4863-4874 
Figure Legends:

Figure 1: Transverse aortic constriction-induced artery remodeling and fibronectin deposition: A. WT mice subjected to sham and TAC surgery. Macroscopic observation revealed thickening of the right carotid artery compared to left and sham carotid arteries. B \& C. Morphometric analysis revealed a significant increase in the vessel area and lumen diameter of the right carotid artery compared with sham or with contralateral left carotids. D. TAC induced deposition of the fibronectin in right carotid artery compared to sham and left carotid. Nuclei were counter stained with DAPI (blue). WT sham mice, $n=5$; WT TAC surgery mice, $n=4$; Statistical analysis: one-way ANOVA with Tukey's post hoc analysis; values are means $\pm \operatorname{SEM}\left({ }^{*} \mathrm{p}<0.05, * * * \mathrm{p}<0.001\right.$, $* * * * \mathrm{p}<0.0001, \mathrm{NS}$, not significant, compared with WT sham.

Figure 2: Transverse aortic constriction-induced artery remodeling in $\alpha 5 / 2$ mice.

WT and $\alpha 5 / 2$ knock in mice at 1 week after TAC surgery. A \& B. Vessel area and lumen diameter in carotid arteries from WT vs. $\alpha 5 / 2$ knock in mice. WT mice, $n=5 ; \alpha 5 / 2$ mice, $n=4$; Statistical analysis: one-way ANOVA with Tukey's post hoc analysis; Values are means \pm SEM; ${ }^{*} \mathrm{p}<0.05, * * * \mathrm{p}<0.001, * * * * \mathrm{p}<0.0001$ compared with WT mice.

Figure 3: Transverse aortic constriction-induced fibronectin deposition and endothelial inflammation.

WT and $\alpha 5 / 2$ knock in mice were subjected to TAC surgery. One-week post-surgery, carotid artery sections were stained for. A. Fibronectin; B. VCAM; C. ICAM; D. CD68; E. NFкB p-65. Nuclei are counter stained with DAPI (blue). WT mice, $n=4 ; \alpha 5 / 2$ knock-in mice, $n=4$; Statistical analysis: one-way ANOVA with Tukey's post hoc analysis; values are means $\pm \mathrm{SEM} ; * * * \mathrm{p}<0.001$ compared with WT mice.

\section{Figure 4. Partial carotid ligation-induced artery remodeling.}

A. WT and $\alpha 5 / 2$ knock in mice subjected to PCL surgery. Briefly, 3 out of 4 branches of the left common carotid artery (left external carotid, internal carotid, and occipital artery) were ligated with suture, while superior thyroid artery was left intact. B. One-week post-surgery, carotid arteries lumen diameter measured. Left carotid artery from WT mice have reduced lumen diameter compared to right carotid arteries form WT mice. In contrast 
to WT mice, integrin $\alpha 5 / 2$ mice showed no change in the lumen area. B. Increase in FN staining in WT mice left carotid compared with $\alpha 5 / 2$ mice. Nuclei are counter stained with DAPI (blue). (WT mice, $n=6 ; \alpha 5 / 2$ knock-in mice, $\mathrm{n}=4)$; Statistical analysis: one-way ANOVA with Tukey's post hoc analysis; Mean \pm SEM $\left(\left({ }^{*} \mathrm{p}<0.05\right.\right.$, $* * \mathrm{p}<0.01$ compared with WT mice)

\section{Figure 5: Partial carotid ligation-induced endothelial inflammation.}

WT and $\alpha 5 / 2$ knock in mice subjected PCL surgery. One-week post-surgery, carotid artery sections stained with following antibodies. A.VCAM; B. ICAM; C. CD68; D. CD45; E. NFKB p-65. Nuclei are counter stained with DAPI (blue). (WT mice, $n=4 ; \alpha 5 / 2$ knock-in mice, $n=4$ ); Statistical analysis: one-way ANOVA with Tukey's post hoc analysis; Mean $\pm \operatorname{SEM}\left(\left({ }^{*} \mathrm{p}<0.05,{ }^{*} \mathrm{p}<0.01\right.\right.$ compared with WT mice $)$

Figure 6: Partial carotid ligation in hypercholesterolemic mice.

8-10 weeks old WT and integrin $\alpha 5 / 2$ mice on the $\mathrm{ApoE}^{-/-}$background were subjected to partial carotid ligation surgery. After the surgery, mice were fed a Western diet for 3 weeks and carotid arteries examined. A. H\&E staining. B. Oil Red O staining. C. CD68 staining. WT;ApoE-/- mice, n=4; $\alpha 5 / 2 ;$ ApoE-/- mice, n=4; Statistical analysis: one-way ANOVA with Tukey's post hoc analysis; Values are means \pm SEM; ${ }^{*} \mathrm{p}<0.05,{ }^{* *} \mathrm{p}<0.01$ compared with WT mice.

\section{Figure 7: Plasma lipids.}

Plasma lipid profiles in WT;ApoE-/- and $\alpha 5 / 2$;ApoE-/- mice: A. Plasma LDL cholesterol; B. total cholesterol; C. Triglycerides. No significant differences were observed. 
A.

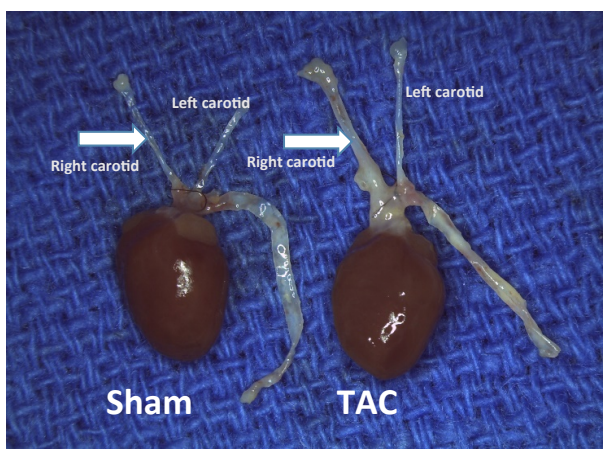

B.

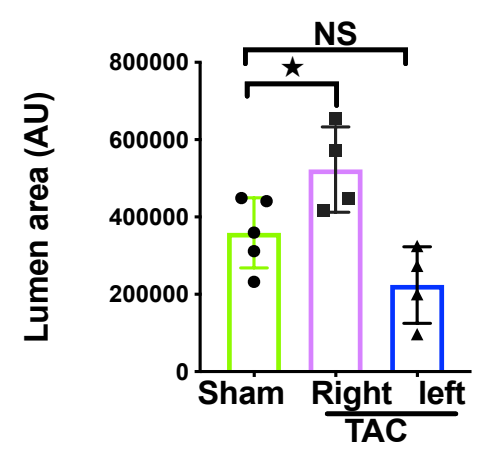

C.

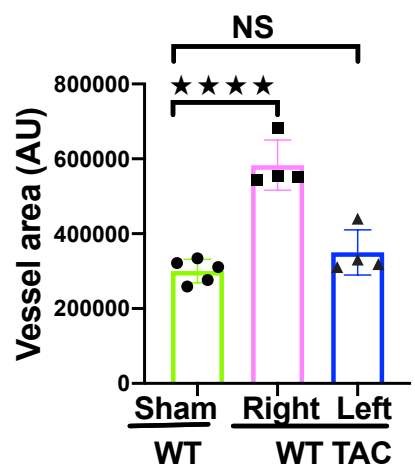

\section{Sham Right carotid}

TAC Right carotid

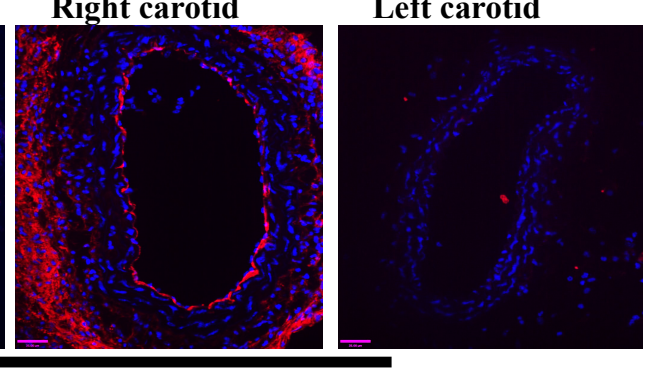

DAPI + Fibronectin

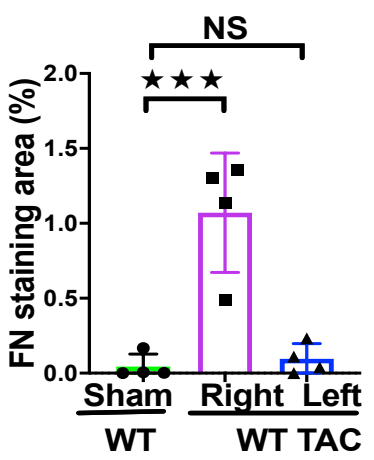

\section{Figure 1}


A.

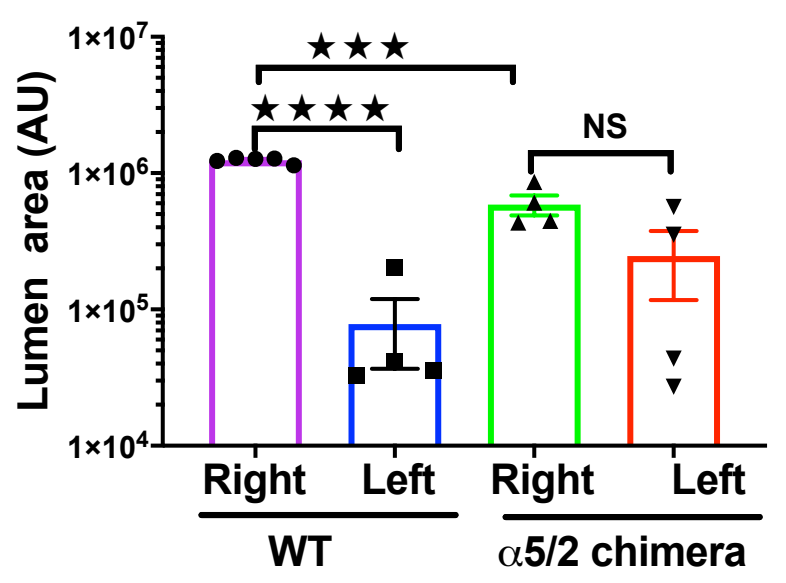

B.

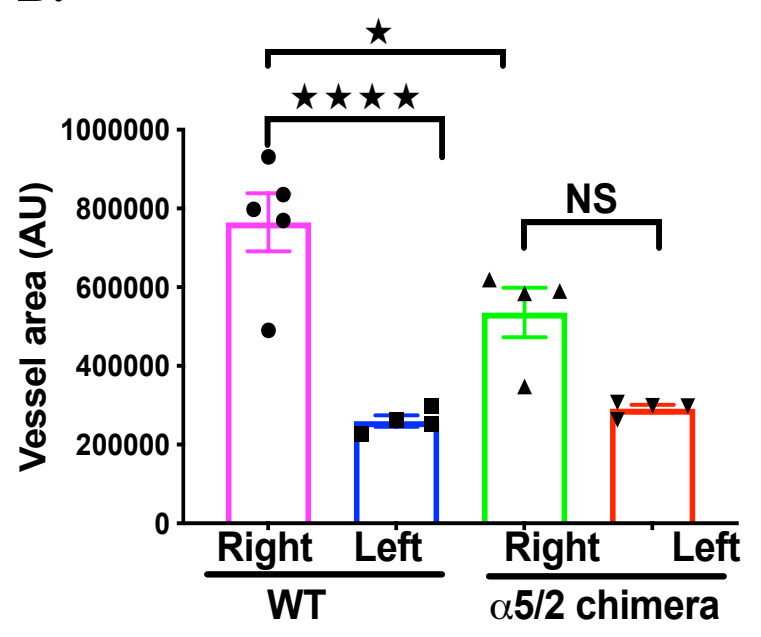

Figure 2 
A.

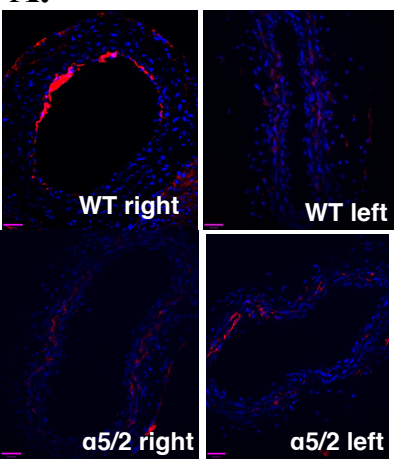

DAPI+Fibronectin
B.
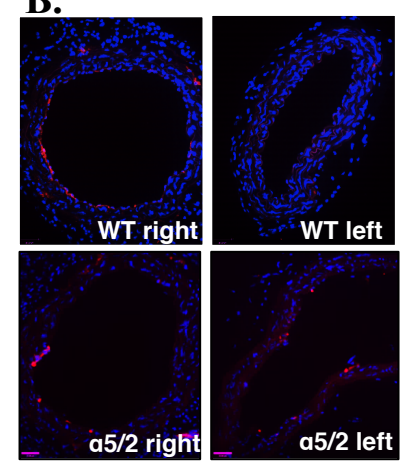

DAPI+VCAM
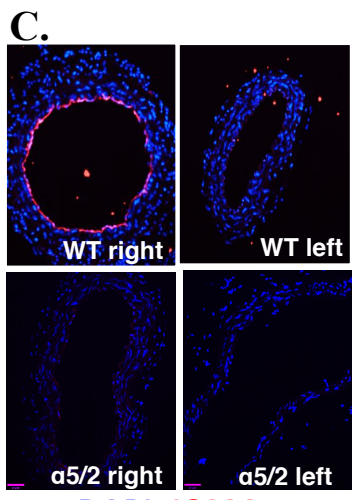

DAPI+ICAM
D.
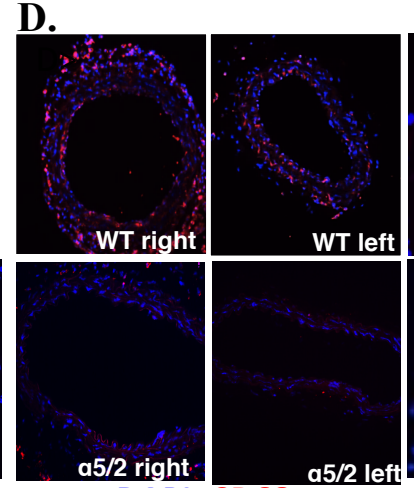

DAPI+CD68
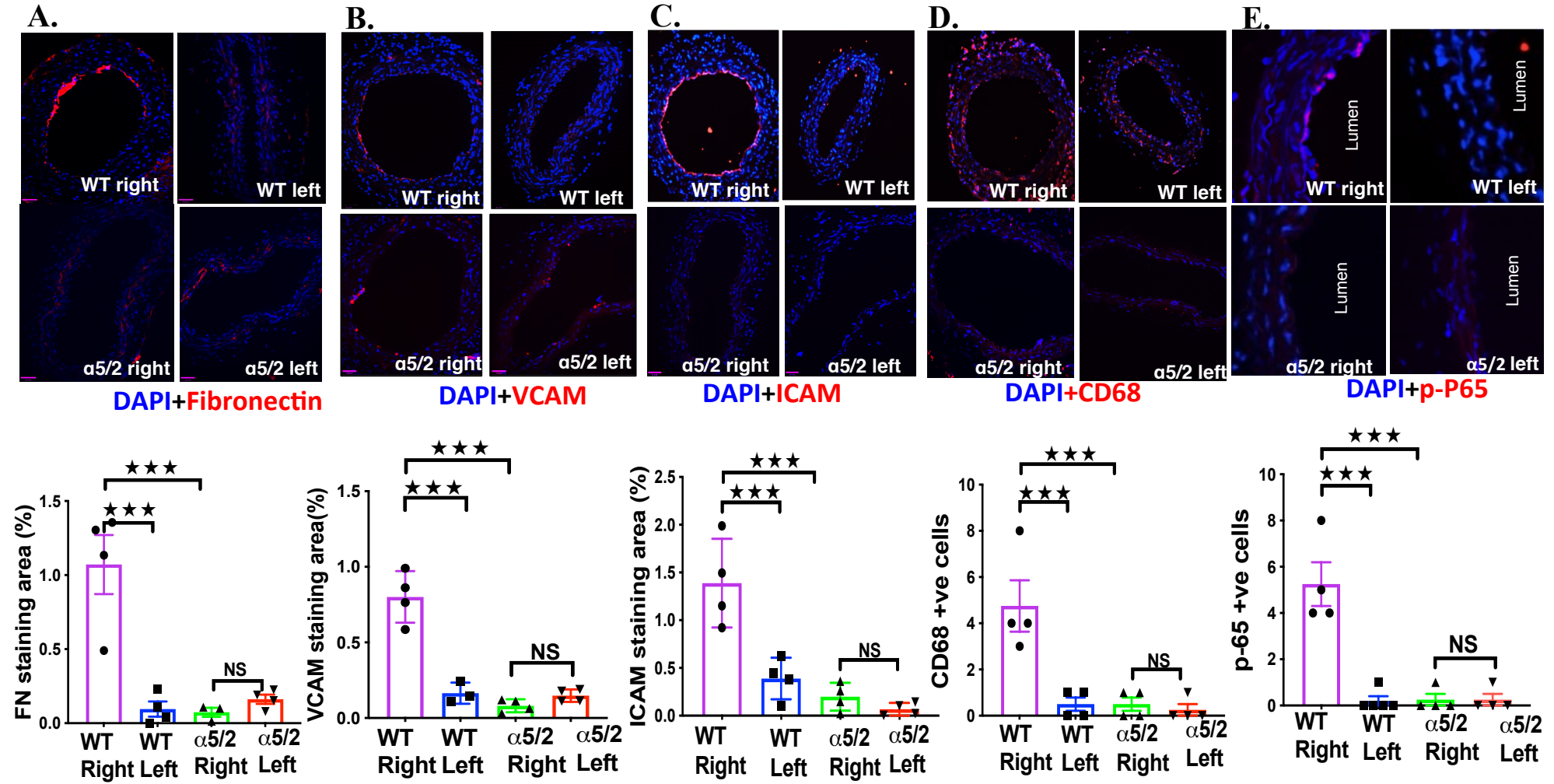

\section{Figure 3}


A.

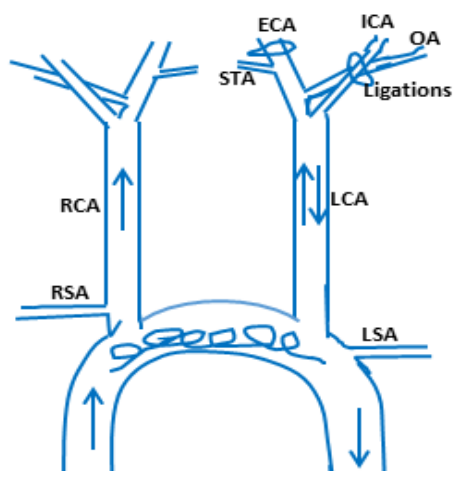

C.
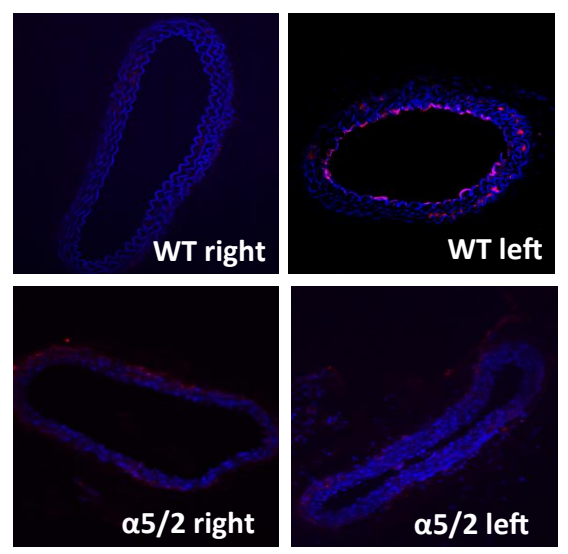

B.
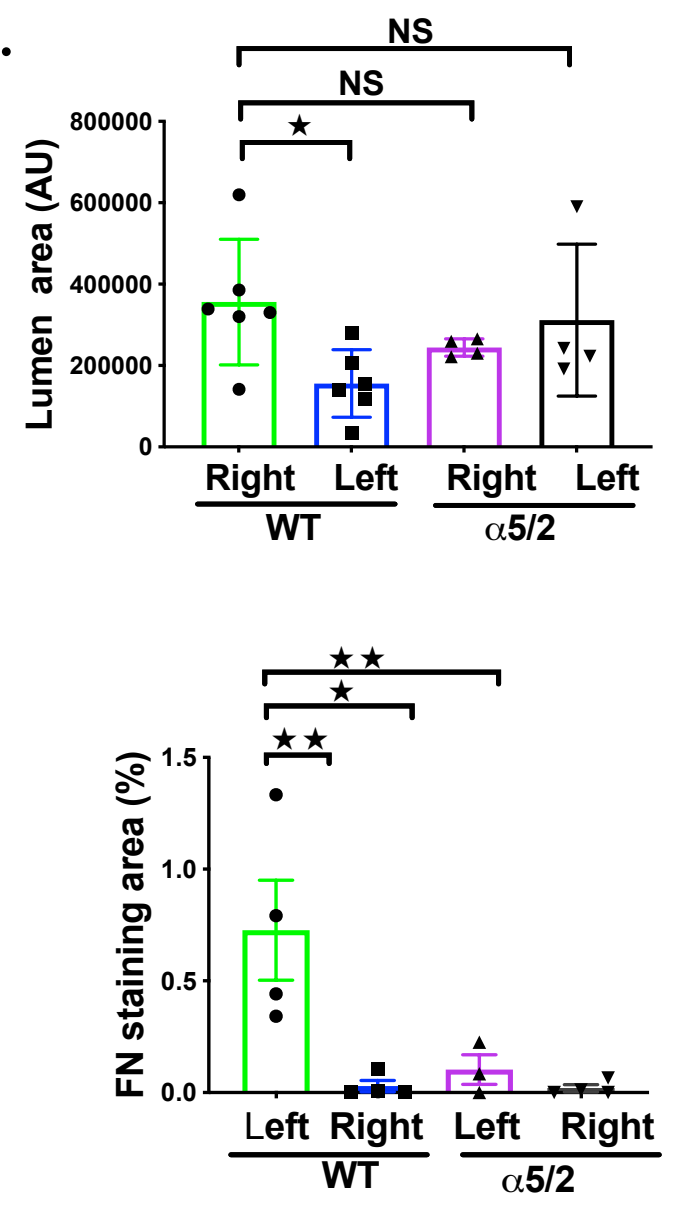

Figure 4 
A.

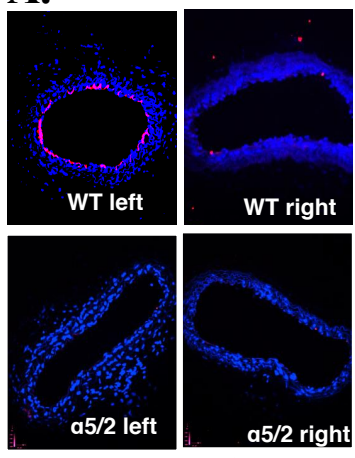

DAPI+VCAM
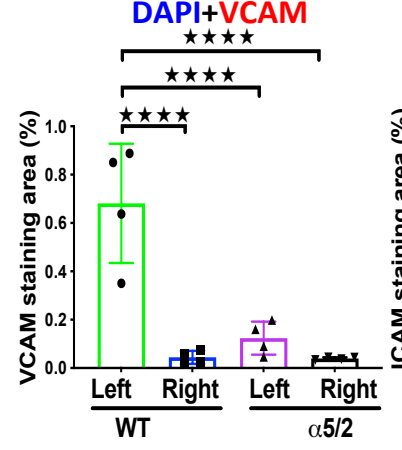

B.

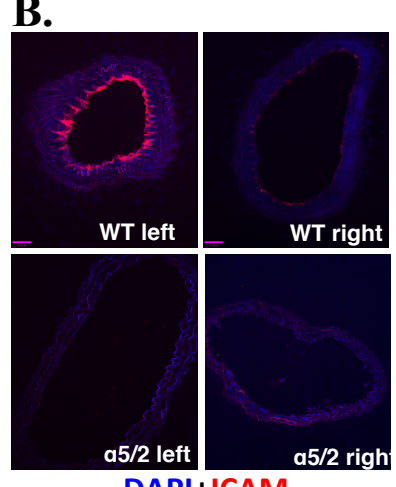

C.

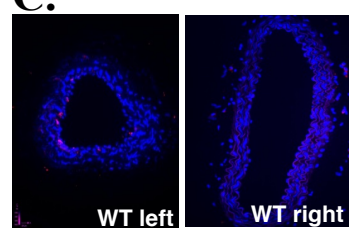

D.

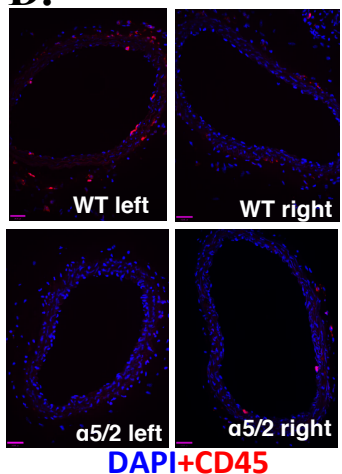

E.
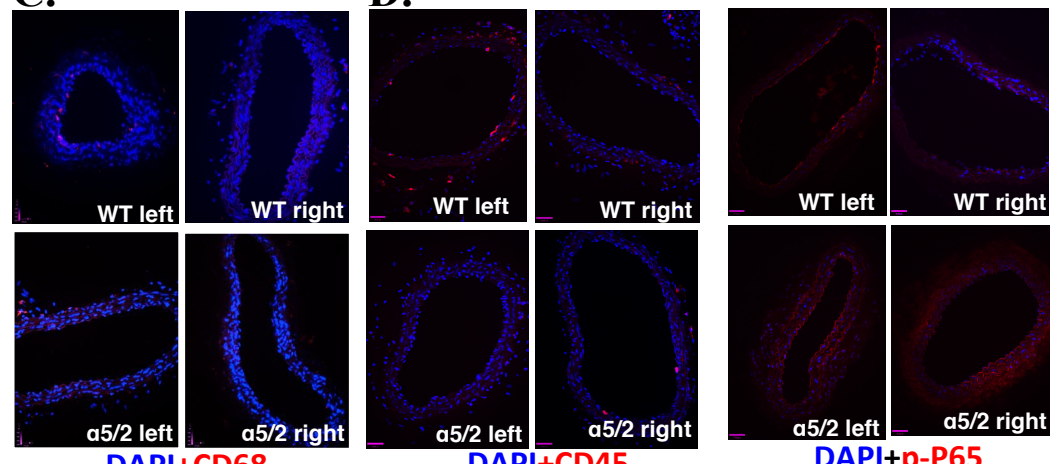

$\mathrm{DAPI}+\mathrm{CD} 68$

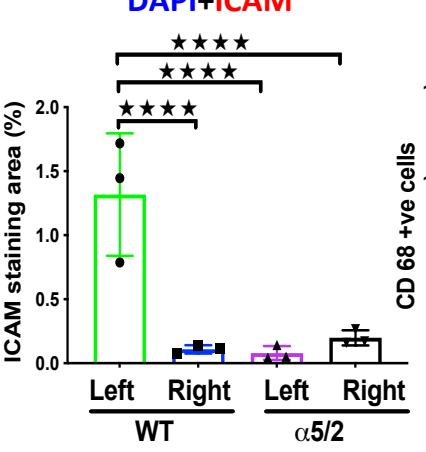

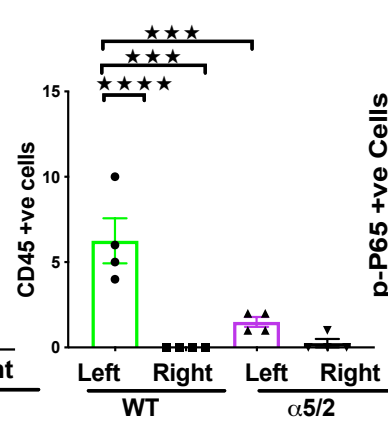

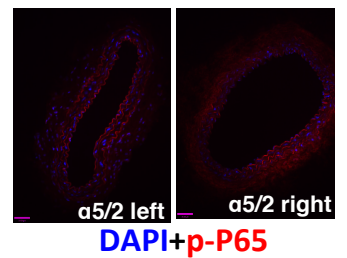

DAPI+p-P65

\section{Figure 5}



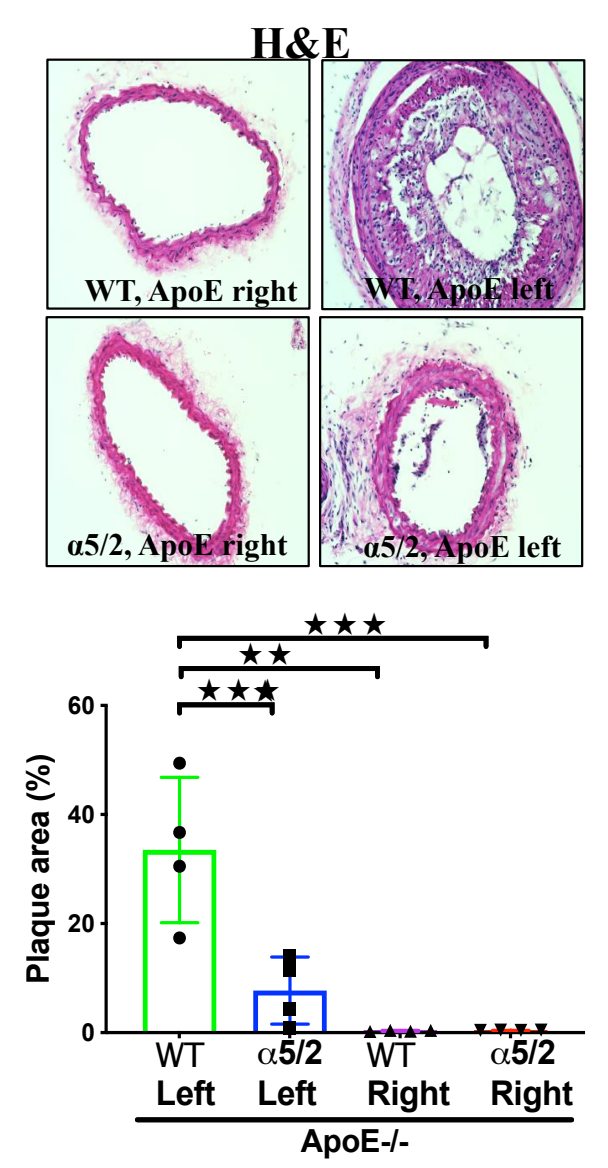
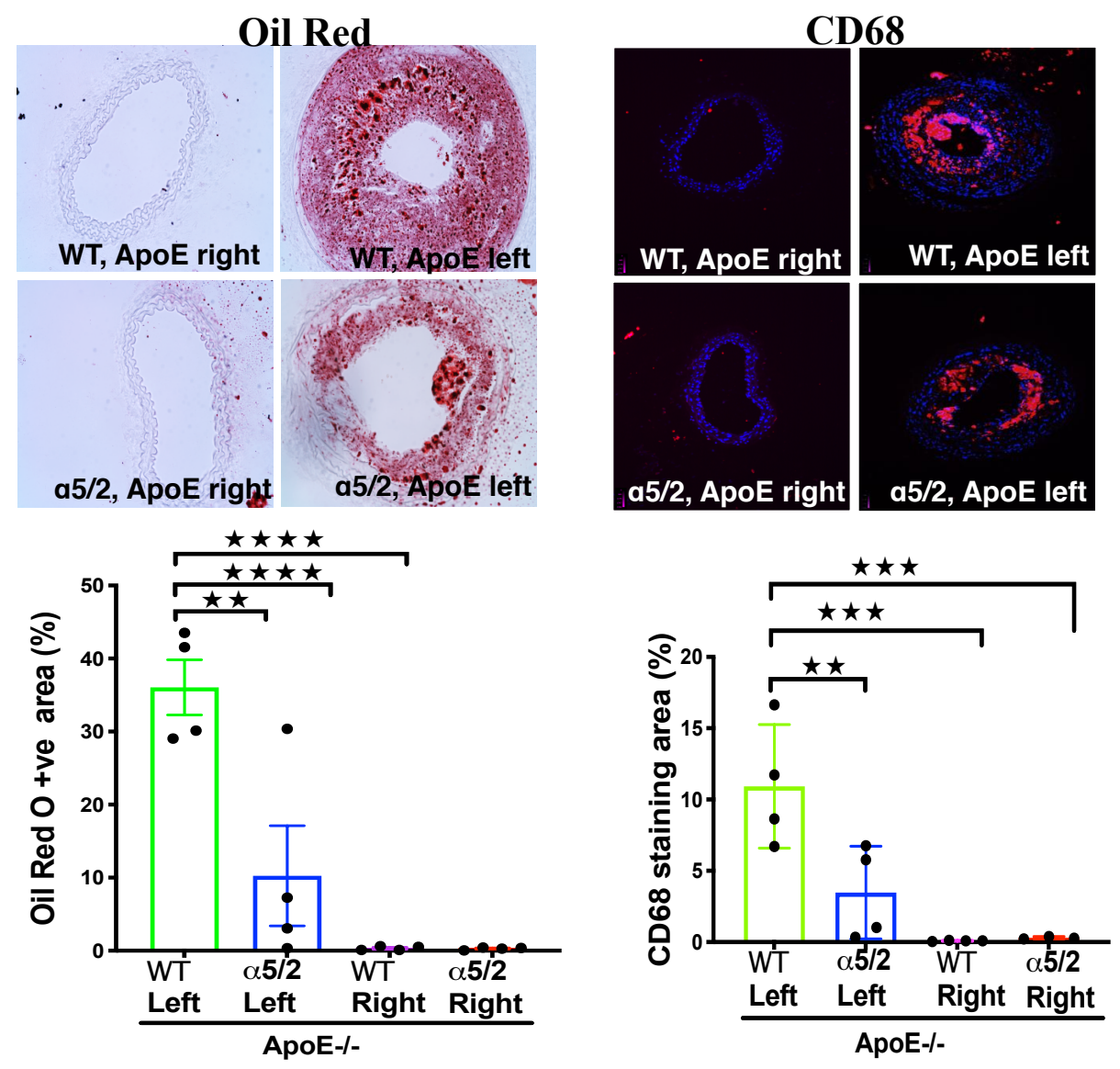

Figure 6 


\section{LDL Cholesterol}

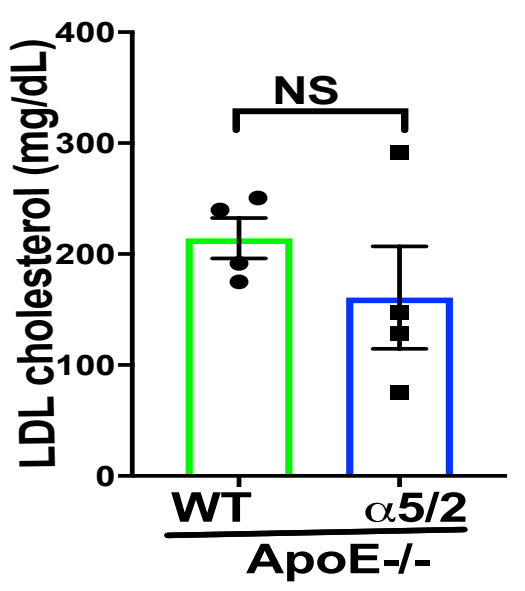

Total Cholesterol

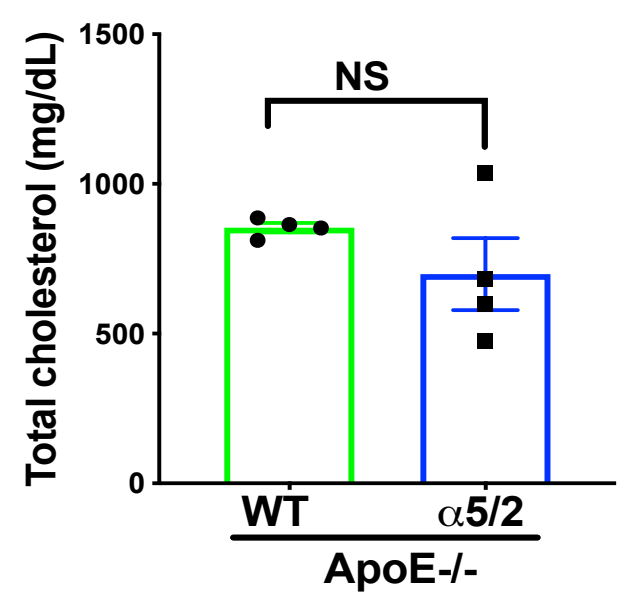

\section{Triglycerides}

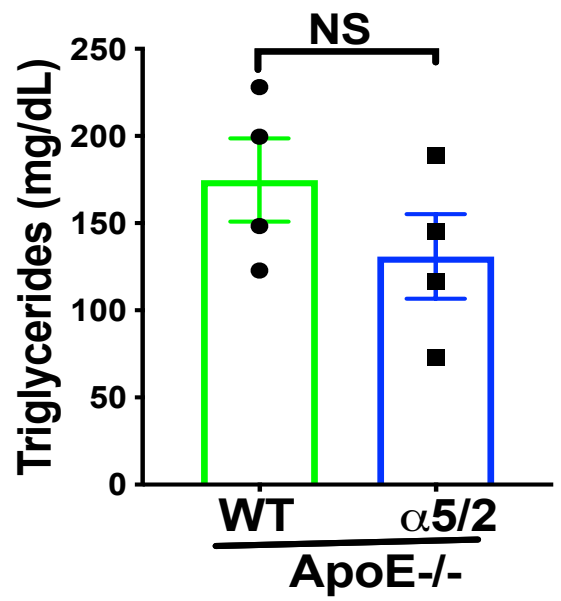

Figure 7 